\title{
A Multiresolution Causal Colour Texture Model
}

\author{
Michal Haindl ${ }^{1,2}$ and Vojtěch Havlíček ${ }^{2}$ \\ 1 Center for Machine Perception, Czech Technical University, \\ 2 Institute of Information Theory and Automation, \\ Academy of Sciences CR, 18208 Prague, Czech Republic \\ \{haindl, havlicek\}@utia.cas.cz
}

\begin{abstract}
An efficient recursive algorithm for realistic colour texture synthesis is proposed. The algorithm starts with spectral factorization of an input colour texture image using the Karhunen-Loeve decorrelation. Single orthogonal monospectral components are further decomposed into a multi-resolution grid and each resolution data are independently modeled by their dedicated simultaneous causal autoregressive random field model (CAR). We estimate an optimal contextual neighbourhood and parameters for each CAR submodel. Finally single synthesized monospectral texture pyramids are collapsed into the fine resolution images and using the inverse Karhunen-Loeve transformation we obtain the required colour texture. The benefit of the multigrid approach is the replacement of a large neighbourhood CAR model with a set of several simpler CAR models which are easy to synthesize and wider application area of these multigrid models capable of reproducing realistic textures for enhancing realism in texture application areas.
\end{abstract}

\section{Introduction}

Virtual reality systems require object surfaces covered with realistic nature-like colour textures to enhance realism in virtual scenes. These textures can be either digitised natural textures or textures synthesized from an appropriate mathematical model. Digitised solid 3D textures are far less convenient, since they involve the 2D digitisation of a large number of cross-sectioned slices through some material. Synthetic textures are more flexible than digitized textures, in that synthetic textures can be designed to have certain desirable properties or meet certain constraints; for example, it can be made smoothly periodic, so that it can be used to fill an infinite texture space without visible discontinuities. While a digitized texture must be stored in a tabular form and evaluated by table lookup, a synthetic texture may be evaluated directly in procedural form. Other texture models applications cover image and video compression, image restoration, image classification and many others.

There are several texture modelling approaches published [6], [5] and some survey articles are also available [4]. Our previous paper [5] introduced a fast multiresolution Markov random field based method. Although this method avoids 
the time consuming Markov chain Monte Carlo simulation so typical for applications of Markov models it requires several approximations. Simultaneous causal autoregressive random fields are appropriate models for texture synthesis not only because they do not suffer with some problems of alternative options (see [3, 44 for details) but they are also easy to analyze as well as to synthesize and last but not least they are still flexible enough to imitate a large set of natural and artificial textures.

Multiple resolution decomposition (MRD) such as Gaussian/Laplacian pyramids, wavelet pyramids or subband pyramids [7], [5] present efficient method for the spatial information compressing. The hierarchy of resolutions provides a transition between pixel-level features and region or global features and hence to model a large variety of possible textures. Unfortunately autoregressive random fields, similarly as the majority of other Markovian types of random field models 2], are not invariant to multiple resolution decomposition (MRD) even for simple MRD like subsampling and the lower-resolution images generally lose their autoregressive property and become ARMA random fields instead. To avoid computationally demanding approximations of an ARMA multigrid random field by an infinite order (i.e., high order in practice) autoregressive random fields we analyze each resolution component independently.

\section{Texture Model}

Modelling general colour texture images requires three dimensional models. If a $3 \mathrm{D}$ data space can be factorized then these data can be modelled using a set of less-dimensional 2D random field models, otherwise it is necessary to use some $3 \mathrm{D}$ random field model. Although full 3D models allows unrestricted spatialspectral correlation modelling its main drawback is large amount of parameters to be estimated and in the case of Markov models (MRF) also the necessity to estimate all these parameters simultaneously. The factorization alternative is attractive because it allows using simpler 2D data models with less parameters (one third in the three-spectral case of colour textures). Unfortunately real data space can be decorrelated only approximately, hence the independent spectral component modelling approach suffers with some loss of image information.

Spectral factorization using the Karhunen-Loeve expansion transforms the original centered data space $\tilde{Y}$ defined on the rectangular $M \times N$ finite lattice $I$ into a new data space with K-L coordinate axes $\bar{Y}$. This new basis vectors are the eigenvectors of the second-order statistical moments matrix (1)

$$
\Phi=E\left\{\tilde{Y}_{r} \tilde{Y}_{r}^{T}\right\}
$$

where the multiindex $r$ has two components $r=\left[r_{1}, r_{2}\right]$, the first component is row and and the second one column index, respectively. The projection of random vector $\tilde{Y}_{r}$ onto the K-L coordinate system uses the transformation matrix

$$
T=\left[u_{1}^{T}, u_{2}^{T}, u_{3}^{T}\right]^{T}
$$


which has single rows $u_{j}$ that are eigenvectors of the matrix $\Phi$.

$$
\bar{Y}_{r}=T \tilde{Y}_{r}
$$

Components of the transformed vector $\bar{Y}_{r}$ (3) are mutually uncorrelated.

Texture modelling does not require computationally demanding MRD approximations (e.g. 2]) because it does not need to propagate information between different data resolution levels. It is sufficient to analyze and subsequently generate single spatial frequency bands without assuming a knowledge of some global multi-grid random field model. We assume colour texture factorized into orthogonal mono-spectral components [5]. These components are further decomposed into a multi-resolution grid and each resolution data are independently modeled by their dedicated CAR. Each one generates a single spatial frequency band of the texture. An analysed texture is decomposed into multiple resolutions factors using Laplacian pyramid and the intermediary Gaussian pyramid. The Gaussian pyramid $\ddot{Y}^{(k)}$ is a sequence of images in which each one is a lowpass down-sampled version of its predecessor where the weighting function (FIR generating kernel) is chosen subject to following constrains:

$$
\begin{array}{rlrl}
w_{s} & =\hat{w}_{s_{1}} \hat{w}_{s_{2}} & \\
\sum_{i} \hat{w}_{i} & =1 \\
\hat{w}_{i} & =\hat{w}_{-i} \\
\hat{w}_{0} & =2 \hat{w}_{1} \quad(l=1)
\end{array}
$$

The solution of above constrains for the reduction factor $3(2 l+1)$ is $\hat{w}_{0}=$ $0.5, \hat{w}_{1}=0.25$ and the FIR equation is now

$$
\ddot{Y}_{r}^{(k)}=\sum_{i, j=-l}^{l} \hat{w}_{i} \hat{w}_{j} \ddot{Y}_{2 r+(i, j)}^{(k-1)} .
$$

The Gaussian pyramid for a reduction factor $n$ is

$$
\ddot{Y}_{r}^{(k)}=\downarrow_{r}^{n}\left(\ddot{Y}^{(k-1)} \otimes w\right) \quad k=1,2, \ldots,
$$

where

$$
\ddot{Y}^{(0)}=\bar{Y},
$$

$\downarrow^{n}$ denotes down-sampling with reduction factor $n$ and $\otimes$ is the convolution operation.

The Laplacian pyramid $\dot{Y}_{r}^{(k)}$ contains band-pass components and provides a good approximation to the Laplacian of the Gaussian kernel. It can be constructed by differencing single Gaussian pyramid layers:

$$
\dot{Y}_{r}^{(k)}=\ddot{Y}_{r}^{(k)}-\uparrow_{r}^{n}\left(\ddot{Y}^{(k+1)}\right) \quad k=0,1, \ldots,
$$


where $\uparrow^{n}$ is the up-sampling with an expanding factor $n$.

Single orthogonal monospectral components are thus decomposed into a multiresolution grid and each resolution data are independently modeled by their dedicated independent Gaussian noise driven autoregressive random field model (CAR) as follows.

The causal autoregressive random field (CAR) is a family of random variables with a joint probability density on the set of all possible realisations $Y$ of the $M \times N$ lattice $I$, subject to following condition:

$$
\begin{aligned}
p\left(Y \mid \gamma, \sigma^{-2}\right)= & (2 \pi)^{-\frac{(M N-1)}{2}}\left|\sigma^{-2}\right|^{\frac{(M N-1)}{2}} \\
& \exp \left\{-\frac{1}{2} \operatorname{tr}\left\{\sigma^{-2}\left(\begin{array}{c}
-I \\
\gamma^{T}
\end{array}\right)^{T} \tilde{V}_{M N-1}\left(\begin{array}{c}
-I \\
\gamma^{T}
\end{array}\right)\right\}\right\},
\end{aligned}
$$

where the following notation is used

$$
\begin{aligned}
\tilde{V}_{r-1} & =\left(\begin{array}{cc}
\tilde{V}_{y(r-1)} & \tilde{V}_{x y(r-1)}^{T} \\
\tilde{V}_{x y(r-1)} & \tilde{V}_{x(r-1)}
\end{array}\right), \\
\tilde{V}_{y(r-1)} & =\sum_{k=1}^{r-1} Y_{k} Y_{k}^{T}, \\
\tilde{V}_{x y(r-1)} & =\sum_{k=1}^{r-1} X_{k} Y_{k}^{T}, \\
\tilde{V}_{x(r-1)} & =\sum_{k=1}^{r-1} X_{k} X_{k}^{T} .
\end{aligned}
$$

The 2D CAR model can be expressed as a stationary causal uncorrelated noise driven $2 \mathrm{D}$ autoregressive process:

$$
Y_{r}=\gamma X_{r}+e_{r}
$$

where $\gamma$ is the parameter vector

$$
\begin{aligned}
& \gamma=\left[a_{1}, \ldots, a_{\eta}\right] \\
& \eta=\operatorname{card}\left(I_{r}^{c}\right)
\end{aligned}
$$

$I_{r}^{c}$ is a causal neighbourhood, $e_{r}$ is a white Gaussian noise with zero mean and a constant but unknown variance $\sigma^{2}$ and $X_{r}$ is a corresponding vector of $Y_{r-s}$ (design vector). 


\section{Parameter Estimation}

The selection of an appropriate CAR model support is important to obtain good results in modelling of a given random field. If the contextual neighbourhood is too small it can not capture all details of the random field. Inclusion of the unnecessary neighbours on the other hand add to the computational burden and can potentially degrade the performance of the model as an additional source of noise.

The optimal Bayesian decision rule for minimizing the average probability of decision error chooses the maximum posterior probability model, i.e., a model $M_{i}$ corresponding to

$$
\max _{j}\left\{p\left(M_{j} \mid Y^{(r-1)}\right)\right\}
$$

where $Y^{(r-1)}$ denotes the known process history

$$
Y^{(r-1)}=\left\{Y_{r-1}, Y_{r-2}, \ldots, Y_{1}, X_{r}, X_{r-1}, \ldots, X_{1}\right\} .
$$

If we assume uniform prior for all tested support sets (models) the solution can be found analytically. The most probable model given past data is the model $M_{i}\left(I_{r, i}^{c}\right)$ for which

$$
\begin{gathered}
i=\arg \max _{j}\left\{D_{j(r-1)}\right\} \\
D_{j(r-1)}=\ln \Gamma\left(\frac{\beta(r)-\eta+2}{2}\right)-\ln \Gamma\left(\frac{\beta(0)-\eta+2}{2}\right) \\
-\frac{1}{2} \ln \left|V_{z(r-1)}\right|-\frac{\beta(r)-\eta+2}{2} \ln \left|\lambda_{(r-1)}\right|
\end{gathered}
$$

where

$$
\begin{gathered}
\beta(r)=\beta(0)+r-1, \\
\beta(0)>1,
\end{gathered}
$$

and

$$
\lambda_{(r)}=V_{y(r)}-V_{x y(r)}^{T} V_{x(r)}^{-1} V_{x y(r)} .
$$

Parameter estimation of a CAR model using the maximum likelihood, the least square or Bayesian methods can be found analytically. The Bayesian parameter estimations of the causal AR model with the normal-gamma parameter prior which maximize the posterior density are:

$$
\hat{\gamma}_{r-1}^{T}=V_{x(r-1)}^{-1} V_{x y(r-1)}
$$

and

$$
\hat{\sigma}_{r-1}^{2}=\frac{\lambda_{(r-1)}}{\beta(r)}
$$



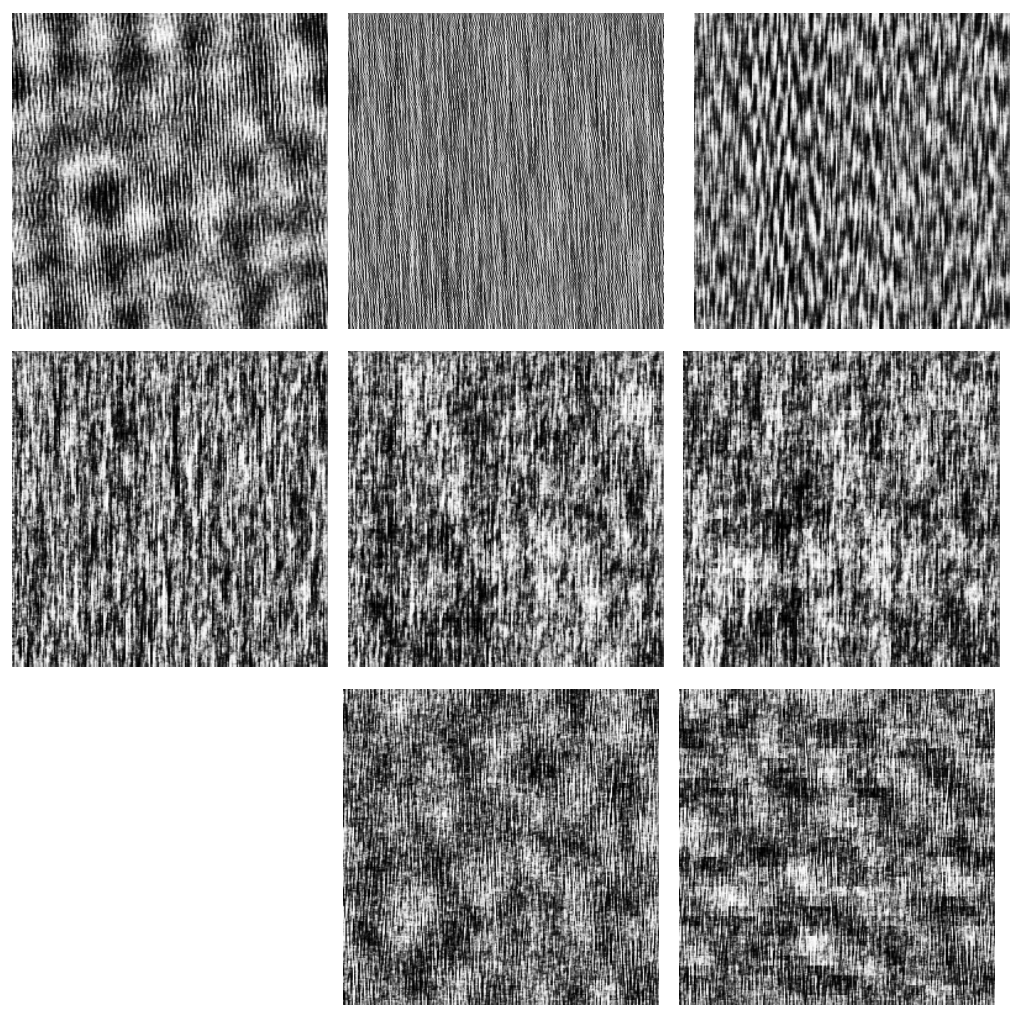

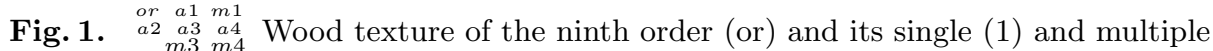
$(2,3,4)$ scales resynthesis using the CAR (a) and MRF (m) models.

where $V_{z(r-1)}=\tilde{V}_{z(r-1)}+V_{z(0)}$ and matrices $V_{z(0)}$ are from parameter prior. The estimates (16), (20), (21) can be also evaluated recursively if necessary.

\section{Model Synthesis}

The CAR model synthesis is very simple and a causal CAR random field can be directly generated from the model equation (12).

Single CAR models synthesize spatial frequency bands of the texture. Each monospectral fine-resolution component is obtained from the pyramid collapse procedure (inversion process to (5), (6) ). Finally the resulting synthesized colour texture is obtained from the set of synthetized monospectral images using the inverse $\mathrm{K}-\mathrm{L}$ transformation:

$$
\tilde{Y}_{r}=T^{-1} \bar{Y}_{r}
$$



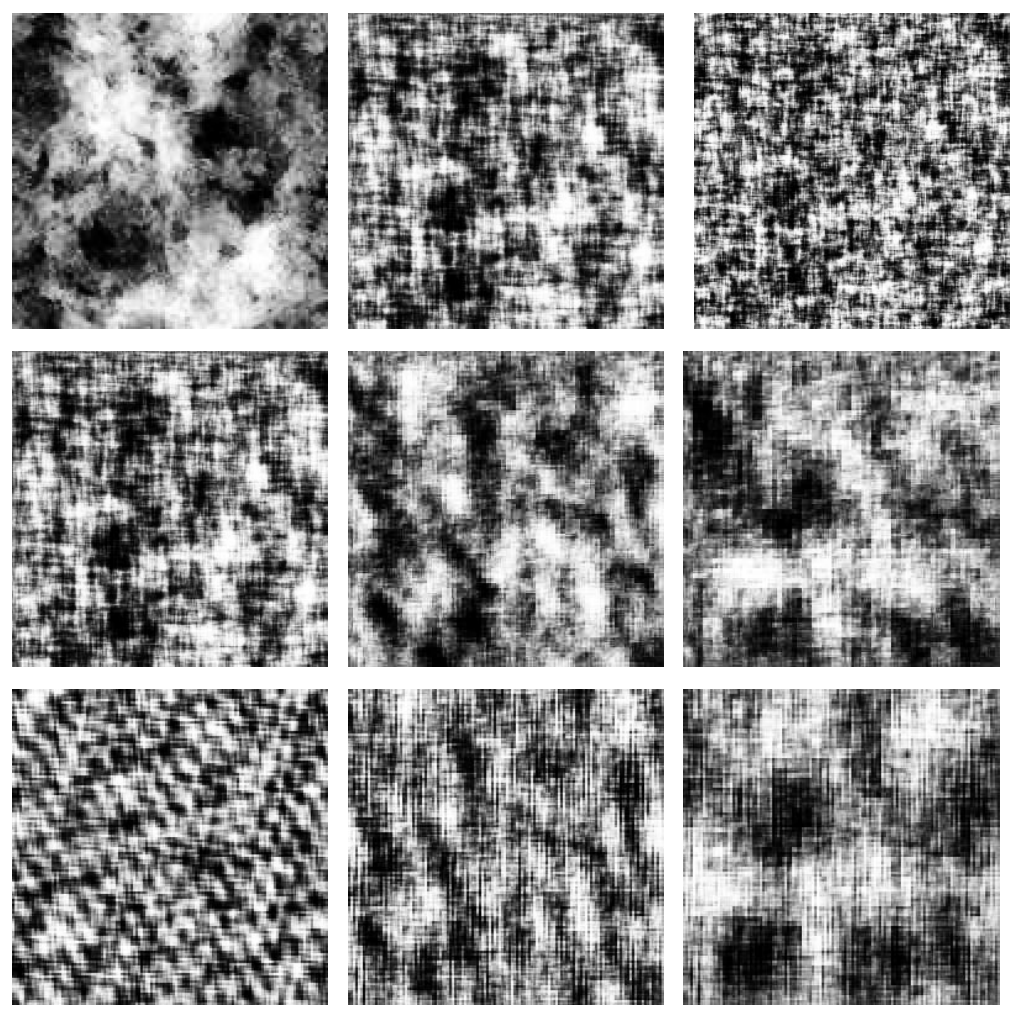

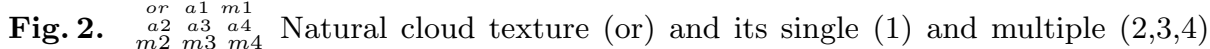
scales resynthesis using the CAR (a) and MRF (m) models.

\section{$5 \quad$ Results}

Two presented natural colour examples (Fig,2 Fig, 3) violates the model stationarity assumption. Nevertheless they are able to demonstrate an advantage of the multiscale approach in texture modelling over single scale models in such unfavourable conditions. Figs, 1, 2 also compare CAR synthesis results with results from the multiscale Markov model [5] synthesis. Fig.1 shows a wood texture synthesized using a ninth order (24 different parameters) MRF model. We tried to resynthesize this texture with inadequate low order CAR and MRF models. The CAR model had only five contextual neighbours and the MRF model was of the second order (4 different parameters). Figs. 1 $1 \mathrm{a} 1, \mathrm{~m} 1), 2(\mathrm{a} 1, \mathrm{~m} 1)$, 3 show unsatisfactory results using the single-scale texture models while these figures simultaneously demonstrate an improvement if we use our presented multi-scale model with two, three or four scale levels, respectively. The second example Fig.2(or) is a natural cloud texture. The texture is non stationary and thus violates the CAR model assumption. Fig[2(a2-a4) show synthesis results for the CAR model while Fig.2(m2-m4) show the second order MRF results for two, 
three or four-scale models, respectively. Both examples Fig,2,Fig 3 are colour textures and they were converted to the grey scale representation only to be printable in the proceedings. The multi-scale models demonstrate their clear superiority over their single-scale counterparts. The colour quality is comparable between single-scale and multi-scale models and it is very good in general.
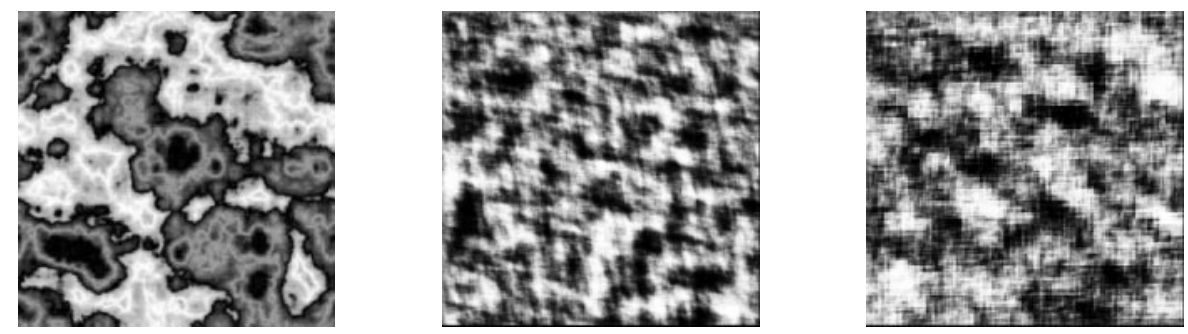

Fig. 3. Natural green marble texture and its single, four-scale resynthesis using the CAR model.

\section{Conclusions}

Our testing results of the algorithm are encouraging. Some synthetic textures reproduce given digitized texture images so that both natural and synthetic texture are visually indiscernible. The multi-scale approach is more robust and allows better results than the single-scale one if the synthesis model is inadequate (lower order model, non stationary texture, etc.). The MRF multiscale model seems to be superior to the causal CAR model for some textures, however the CAR model synthesis is much faster than the MRF model synthesis. The CAR model is better suited for real time or web distributed texture modelling applications. The proposed method allows large compression ratio for transmission or storing texture information while it has very moderate computation complexity.

\section{Acknowledgements}

This research was supported by the GAČR grant no. 102/00/0030 Texture Modelling and partially funded by the Hewlett Packard project no. ISE-86L8-13 and the GAČR grant no. 106/00/0030 Computer aided quantitative fractography of fatique failures. 


\section{References}

1. Besag, J.: Spatial Interaction and the Statistical Analysis of Lattice Systems. J. Royal Stat. Soc. B-36 (1974) 192-236

2. Gidas, B.: A renormalization Group Approach to Image Processing problems. IEEE Trans. Pattern Anal. Mach. Int. 11 (1989) 164-180

3. Haindl, M.: Texture Synthesis. Research Report no. CS-R9139, Centrum voor Wiskunde en Informatica, Amsterdam, 1991

4. Haindl, M.: Texture Synthesis. CWI Quarterly 4 (1991) 305-331

5. Haindl, M., Havlíček, V.: Multiresolution Colour Texture Synthesis. In: Dobrovodský, K. (ed.): Proceedings 7th Int. Workshop RAAD'98, ISBN: 80-967962-7-5, ASCO Art \& Science, Bratislava (1998) 339 - 344

6. Kashyap, R.L.: Analysis and Synthesis of Image Patterns by Spatial Interaction Models. In: Progress in Pattern Recognition 1, (Eds.) L.N. Kanal A.Rosenfeld, Elsevier, North-Holland (1981)

7. Rosenfeld, A. (ed.): Multiresolution Image Processing and Analysis. SpringerVerlag, Berlin Heidelberg New York (1984) 